\title{
A NOTE ON GENERIC SUBSETS OF DEFINABLE GROUPS
}

\author{
MÁRIO J. EDMUNDO AND G. TERZO
}

\begin{abstract}
In this paper we generalize the theory of generic subsets of definably compact definable groups to arbitrary o-minimal structures. This theory is a crucial part of the solution to Pillay's conjecture connecting definably compact definable groups with Lie groups.
\end{abstract}

Date: June 24, 2011

2000 Mathematics Subject Classification. 03C64; $20 \mathrm{E} 99$.

The first author was supported by Fundação para a Ciência e a Tecnologia, Financiamento Base 2008 - ISFL/1/209. This work is part of the FCT project PTDC/MAT/101740/2008. Keywords and phrases: O-minimal structures, definable groups. 


\section{INTRODUCTION}

In [21] Pillay introduced the following very interesting conjecture connecting definably compact definable groups with Lie groups which in the last years has led to important developments in the model theory and geometry of o-minimal structures:

Pillay's Conjecture [21]: Let $G$ be a definably compact, definably connected, definable group in a sufficiently saturated o-minimal structure. Then:

(1) $G$ has a minimal type-definable normal subgroup of bounded index, call it $G^{00}$.

(2) $G / G^{00}$, equipped with the Logic topology, is isomorphic, as a topological group, to compact real Lie group.

(3) $\operatorname{dim} G=\operatorname{dim} G / G^{00}$.

Pillay's conjecture has now been proved in three different situations: in ominimal expansions of fields by Hrushovski, Peterzil and Pillay [13], in linear ominimal expansions of ordered groups by Eleftheriou and Starchenko [12] and in non-linear semi-bounded o-minimal expansions of groups by Peterzil [14]. So Pillay's conjecture holds in arbitrary o-minimal expansions of groups. In all of the three cases above the conjecture is a consequence of the following two crucial ingredients (after the paper [1] by Berarducci, Otero, Peterzil and Pillay where the existence of $G^{00}$ with properties (1) and (2) is proved):

(i) the model theory of generic subsets of definably compact definable groups from [17] together with the heavy model theory of definable ameanable groups from [13]; (ii) the computation of $m$-torsion subgroups of abelian definably compact definable groups ([7], [12] and [14] respectively in each one of the three cases).

In this paper we generalize the theory of generic subsets of definably compact definable groups, which is presented in [17] in an o-minimal expansion of a real closed field, to an arbitrary o-minimal structure. The computation of $m$-torsion subgroups of abelian definably compact definable groups in the field case uses the o-minimal singular cohomology theory from [10] and [22], in the linear case this is obtained by a structure theorem for such definable groups ([12]) and in the non-linear semibounded case by reduction to the field case ([14]). With a good cohomology theory in arbitrary o-minimal structures which generalizes the o-minimal singular cohomology in o-minimal expansion of real closed fields ([10] and [22]) one could obtain a uniform proof of the computation of $m$-torsion subgroups of abelian definably compact definable groups in arbitrary o-minimal structures which would include the three cases above. The authors already have made significant advances in this direction building on previous joint work with other authors ([6], [8] and [9]).

In the paper [17] the authors work in a saturated o-minimal structure expanding a real closed field, and develop a theory of generic subsets based on the work by A. Dolich [3]. This assumption is necessary there since the theory presented by them requires the definable groups to be affine in order to apply [17, Theorem 2.1]. (See the explanation in [17, Subsection 1.1]). In the case of an o-minimal expansion of a real closed field every regular definable space is affine up to definable homeomorphism. (See [2] for the definition). However, by a trick due to Peterzil and Eleftheriou ([14, Section 8$])$, this theory of generic sets also works for arbitrary 
definably compact definable groups in saturated o-minimal expansions of ordered groups, even though in this case, there are definable groups which are not affine ([11]). Here we point out that this trick can be generalized to definably compact definable groups in arbitrary saturated o-minimal structures.

\section{Definable normality in Definable Groups}

Let $\mathcal{M}=\langle M,<, \ldots\rangle$ be an o-minimal structure. By definable we will mean definable in $\mathcal{M}$ possibly with parameters. A definable group is a group whose underlying set is a definable set and the graphs of the group operations are definable sets. By [20] a definable group has unique definable manifold structure making it into a topological group. All topological notions in a definable group, unless otherwise stated, will be with respect to the unique definable manifold structure. A definable group is definably connected if it is a definably connected definable manifold, equivalently if it has no proper definable subgroups of finite index ([20]). A definable group $G$ is definably compact if for every continuous definable map $\alpha:(a, b) \subseteq M \cup\{-\infty,+\infty\} \longrightarrow G$ the limits $\lim _{t \rightarrow a^{+}} \alpha(t)$ and $\lim _{t \rightarrow b^{-}} \alpha(t)$ exist in $G([15])$. For the basic theory of definable groups we refer the reader to [5], [16], [18], [19] and [20].

Here we show that every definable group is definably locally compact and every definably compact definable subset of a definable group is definably normal.

Notation: Let $H \subseteq M^{k}$ be a definable group of dimension $n$ with definable charts $\left\langle U_{i}, \phi_{i}\right\rangle(i=1, \ldots, l)$ for its unique definable manifold structure. Let $U=U_{i}$ be such that the identity element $e_{H}$ of $H$ is in $U_{i}$ and set $\phi=\phi_{i}$. Let $\phi\left(e_{H}\right)=\left\langle e_{1}, \ldots, e_{n}\right\rangle$ and for each $j=1, \ldots, n$, take $J_{j}=\left(d_{j}^{-}, d_{j}^{+}\right) \subseteq M$ to be an open (definable) interval such that $e_{j} \in J_{j}$ and $\Pi_{j=1}^{n} J_{j} \subseteq \phi(U)$. Let $O=\phi^{-1}\left(\Pi_{j=1}^{n} J_{j}\right)$ which is an open definable neighborhood of $e_{H}$ in $H$.

For each $j=1, \ldots, n$, let $J_{j}^{-}=\left\{x \in J_{j}: x<e_{j}\right\}$ and $J_{j}^{+}=\left\{x \in J_{j}: e_{j}<x\right\}$. For $\delta=\left\langle\delta_{1}^{-}, \delta_{1}^{+}, \ldots, \delta_{n}^{-}, \delta_{n}^{+}\right\rangle \in \Pi_{j=1}^{n}\left(J_{j}^{-} \times J_{j}^{+}\right)$, let

$$
O^{\delta}=\left\{x \in O: \phi(x)=\left\langle z_{1}, \ldots, z_{n}\right\rangle \text { and } \delta_{j}^{-}<z_{j}<\delta_{j}^{+} \text {for all } j\right\}
$$

and

$$
\bar{O}^{\delta}=\left\{x \in O: \phi(x)=\left\langle z_{1}, \ldots, z_{n}\right\rangle \text { and } \delta_{j}^{-} \leq z_{j} \leq \delta_{j}^{+} \text {for all } j\right\} .
$$

If $D$ is a definable subset of $H$, we consider

$$
O^{\delta}(D)=\cup\left\{d O^{\delta}: d \in D\right\}
$$

and

$$
\bar{O}^{\delta}(D)=\cup\left\{d \bar{O}^{\delta}: d \in D\right\} .
$$

We have that $O^{\delta}(D)$ is open definable neighborhood of $D$ in $H$ and $O^{\delta}(D) \subseteq \bar{O}^{\delta}(D)$.

Lemma 2.1. Let $H$ be a definable group. If $D$ is a definably compact definable subset of $H$, then $\bar{O}^{\delta}(D)$ is a definably compact definable neighborhood of $D$ in $H$. 
Proof. Let $\alpha:(b, c) \subseteq M \longrightarrow \bar{O}^{\delta}(D)$ be a continuous definable map. Since $G$ has definable choice ([5, Theorem 7.2]), so thus $\bar{O}^{\delta}(D)$. Hence, there is a continuous definable map $\beta:(b, c) \subseteq M \longrightarrow D$ such that for all $t \in(b, c)$ we have $\alpha(t) \in \bar{O}^{\delta}(\beta(t))$. Therefore we have a continuous definable map $\psi:(b, c) \subseteq M \longrightarrow$ $\Pi_{j=1}^{n}\left[\delta_{j}^{-}, \delta_{j}^{+}\right]$given by $\psi(t)=\phi\left(\beta^{-1}(t) \alpha(t)\right)$. Since $D$ is definably compact, there is $x \in D$ such that $\lim _{t \rightarrow c} \beta(t)=x$. On the other hand, since $\Pi_{j=1}^{n}\left[\delta_{j}^{-}, \delta_{j}^{+}\right]$is definably compact, there is $y \in \Pi_{j=1}^{n}\left[\delta_{j}^{-}, \delta_{j}^{+}\right]$such that $\lim _{t \rightarrow c} \psi(t)=y$. By continuity we have

$$
\lim _{t \rightarrow c} \alpha(t)=x \phi^{-1}(y) \in \bar{O}^{\delta}(x) \subseteq \bar{O}^{\delta}(D),
$$

showing that $\bar{O}^{\delta}(D)$ is definably compact.

We say that a definable group $G$ is definably locally compact if for every definably compact definable subset $K$ of $G$ and every open definable neighborhood $U$ of $K$ in $G$, there exists a definably compact neighborhood of $K$ in $U$.

Proposition 2.2. Let $G$ be a definable group. Then for every definably compact definable subset $K$ of $G$ and every open definable neighborhood $U$ of $K$ in $G$, there exists a definably compact neighborhood of $K$ in $U$ of the form $\bar{O}^{\epsilon}(K)$. In particular, $G$ is definably locally compact.

Proof. We prove the result by induction on the $\operatorname{dim} K$. Clearly the result is true for $\operatorname{dim} K=0$ and suppose it is true for every definably compact subset $L$ with $\operatorname{dim} L<\operatorname{dim} K$. Let $K$ be a definably compact definable subset of $G$ and $U$ an open definable neighborhood of $K$ in $G$. Since $\phi(O)$ has definable choice, there exist a definable map

$$
g: K \longrightarrow \Pi_{j=1}^{n}\left(J_{j}^{-} \times J_{j}^{+}\right): a \mapsto\left\langle g_{1}^{-}(a), g_{1}^{+}(a), \ldots, g_{n}^{-}(a), g_{n}^{+}(a)\right\rangle
$$

such that $U_{a}=a \phi^{-1}\left(\Pi_{j=1}^{n}\left(g_{j}^{-}(a), g_{j}^{+}(a)\right)\right)$ is an open definable neighborhood of $a$ in $G$ contained in $U$. The definable subset of $K$ on which $g$ is not continuous is a definable set of dimension strictly less than $\operatorname{dim} K$. Let $L$ be the closure of this set in $K$. Then $\operatorname{dim} L<\operatorname{dim} K([2$, Chapter $4,(1.8)])$ and $L$ is a definably compact subset of $G$ contained in $U$. So, by the induction hypothesis, there exist a definably compact neighborhood in $U$ of $L$ of the form $\bar{O}^{\eta}(L)$.

Take $L^{\prime}=K \cap\left(G \backslash O^{\eta}(L)\right)$. Then $L^{\prime}$ is definably compact and $g_{\mid L^{\prime}}: L^{\prime} \longrightarrow$ $\Pi_{j=1}^{n}\left(J_{j}^{-} \times J_{j}^{+}\right)$is continuous. Fix $j \in\{1, \ldots, n\}$. Then for all $a \in L^{\prime}$ we have $g_{j}^{-}(a)<e_{j}<g_{j}^{+}(a)$. We show that there exist $d_{j}^{-}<e_{j}<d_{j}^{+}$such that $g_{j}^{-}(a) \leq$ $d_{j}^{-}<d_{j}^{+} \leq g_{j}^{+}(a)$ for all $a \in L^{\prime}$. Suppose there is no such $d_{j}^{+}$. Then for all $e_{j}<s$ there exists $a \in L^{\prime}$ such that $e_{j}<g_{j}^{+}(a)<s$. Since $G$ has definable choice ([5, Theorem 7.2]), so thus $L^{\prime}$. Hence, there is a definable map $\alpha_{j}^{+}:\left(e_{j}, c_{j}\right) \subseteq J_{j}^{+} \longrightarrow L^{\prime}$ such that for all $e_{j}<t<c_{j}$ we have $e_{j}<g_{j}^{+}\left(\alpha_{j}^{+}(t)\right)<t$. By o-minimality we may assume that $\alpha_{j}^{+}$is continuous. Since $L^{\prime}$ is definably compact there is $e \in L^{\prime}$ such that $\lim _{t \rightarrow e_{j}} \alpha_{j}^{+}(t)=e$. So $g_{j}^{+}(e)=g_{j}^{+}\left(\lim _{t \rightarrow e_{j}} \alpha_{j}^{+}(t)\right)=\lim _{t \rightarrow e_{j}} g_{j}^{+}\left(\alpha_{j}^{+}(t)\right)=e_{j}$ which is a contradiction. Similarly, $d_{j}^{-}$exists. By construction, for all $a \in L^{\prime}$, $a \phi^{-1}\left(\Pi_{j=1}^{n}\left(d_{j}^{-}, d_{j}^{+}\right)\right)$is an open definable neighborhood of $a$ in $G$ contained in $U$. Hence $O^{d}\left(L^{\prime}\right) \subseteq U$ where $d=\left\langle d_{1}^{-}, d_{1}^{+}, \ldots, d_{n}^{-}, d_{n}^{+}\right\rangle$. Let $\delta=\left\langle\delta_{1}^{-}, \delta_{1}^{+}, \ldots, \delta_{n}^{-}, \delta_{n}^{+}\right\rangle$ 
where for each $j, d_{j}^{-}<\delta_{j}^{-}<e_{j}<\delta_{j}^{+}<d_{j}^{+}$. Now take $\epsilon=\left\langle\epsilon_{1}^{-}, \epsilon_{1}^{+}, \ldots, \epsilon_{n}^{-}, \epsilon_{n}^{+}\right\rangle$ where for each $j, \epsilon_{j}^{-}=\max \left\{\delta_{j}^{-}, \eta_{j}^{-}\right\}$and $\epsilon_{j}^{+}=\min \left\{\delta_{j}^{+}, \eta_{j}^{+}\right\}$. Then by Lemma 2.1, $\bar{O}^{\epsilon}(K)=\bar{O}^{\epsilon}(L) \cup \bar{O}^{\epsilon}\left(L^{\prime}\right)$ is a definably compact definable neighborhood of $K$ in $U$.

Corollary 2.3. Let $G$ be a definable group. If $K$ and $C$ are disjoint closed definable subsets of $G$ with $K$ definably compact, then there exist open, disjoint definable neighborhoods $O^{\delta}(K)$ and $G \backslash \bar{O}^{\epsilon}(K)$ of $K$ and $C$ respectively in $G$ for some $\epsilon$ and $\delta$. In particular, if $\Omega$ is a closed definable subset of $G$, then:

(1) $\Omega$ is definably regular;

(2) If $\Omega$ a definably compact, then $\Omega$ is definably normal. In fact, any two disjoint closed definable subsets $A$ and $B$ of $\Omega$ can be separated by open, disjoint, definable neighborhoods of the form $O^{\delta}(A) \cap \Omega$ and $O^{\eta}(B) \cap \Omega$.

Proof. Recall that $\Omega$ is definably regular if for every closed definable subset $C$ of $\Omega$ and $a$ a point of $\Omega$ not in $C$, then there exist open, disjoint definable neighborhoods $U$ and $V$ of $a$ and $C$ respectively in $\Omega$. We say that $\Omega$ is definably normal if for every pair $K$ and $C$ of closed, disjoint definable subsets of $\Omega$ there exist open, disjoint definable neighborhoods $U$ and $V$ of $K$ and $C$ respectively in $\Omega$. So, by Proposition 2.2, there exists a definably compact neighborhood of $K$ in $G \backslash C$ of the form $\bar{O}^{\epsilon}(K)$. For the statement it is enough to choose $\delta=\left\langle\delta_{1}^{-}, \delta_{1}^{+}, \ldots, \delta_{n}^{-}, \delta_{n}^{+}\right\rangle$where for each $j, \epsilon_{j}^{-}<\delta_{j}^{-}<e_{j}<\delta_{j}^{+}<\epsilon_{j}^{+}$, and take $U=O^{\delta}(K)$ and $V=G \backslash \bar{O}^{\epsilon}(K)$.

Corollary 2.4 (Shrinking lemma). Let $G$ be a definable group. If $\Omega$ is a definably compact definable subset of $G$ and $U_{1}, \ldots, U_{l}$ are open definable subsets of $G$ such that $\Omega=\bigcup_{i}\left(U_{i} \cap \Omega\right)$, then there are open definable subsets $V_{i} \subseteq U_{i}$ with $i=1, \ldots, l$ such that $\overline{V_{i}} \subseteq U_{i}$ and $\Omega=\bigcup_{i}\left(V_{i} \cap \Omega\right)$. Moreover, for each $i=1, \ldots, l$, we have $V_{i}=O^{\delta_{i}}\left(A_{i}\right)$ for some $\delta_{i}$ where the closed definable subsets $A_{i}$ of $\Omega$ are given inductively by $A_{i}=\Omega \backslash\left(\bigcup_{m<i} V_{m} \cup \bigcup_{j=i+1}^{l} U_{j}\right)$.

Proof. This follows from the fact that $\Omega$ is definably normal (Corollary 2.3 (2)) and the shrinking lemma whose affine version is [2, Chapter 6, Lemma 3.6]. In fact, assume inductively that $V_{i} \subseteq U_{i}$ has been defined for $i=1, \ldots, k(k<l)$ such that $V_{i}$ is a definable open subset of $G, \overline{V_{i}} \subseteq U_{i}$ and $V_{1}, \ldots, V_{k}, U_{k+1}, \ldots, U_{l}$ cover $\Omega$. Then apply Corollary 2.3 (2) to the following two disjoint closed definable subsets of $\Omega$ :

$$
B=\Omega \backslash U_{k+1} \text { and } C=\Omega \backslash\left(\bigcup_{m \leq k} V_{m} \cup \bigcup_{j=k+2}^{l} U_{j}\right) .
$$

\section{Generic subsets of Definable groups}

Here we prove the main results of the paper. We start with the generalization of [14, Lemma 7.1] from semi-bounded o-minimal expansions of groups ([4]) to arbitrary o-minimal structures.

Lemma 3.1. Let $G$ be a definable group of dimension $n$ with definable charts $\left\langle U_{i}, \phi_{i}\right\rangle$ with $i=1, \ldots$, l. If $\Omega$ is a definably compact definable subset of $G$, then 
there are open definable subsets $V_{i} \subseteq U_{i}$ of $G$, with $i=1, \ldots, l$, such that $\overline{V_{i}} \subseteq U_{i}$, $\Omega=\bigcup_{i}\left(V_{i} \cap \Omega\right)$ and, for each $i, \phi_{i}\left(\overline{V_{i}} \cap \Omega\right)$ a closed and bounded definable subset of $M^{n}$. Furthermore, for each $i=1, \ldots, l$, we have $V_{i}=O^{\delta_{i}}\left(A_{i}\right)$ for some $\delta_{i}$ where the closed definable subsets $A_{i}$ of $\Omega$ are given inductively by $A_{i}=\Omega \backslash\left(\bigcup_{m<i} V_{m} \cup\right.$ $\left.\bigcup_{j=i+1}^{l} U_{j}\right)$.

Proof. Suppose that $G \subseteq M^{k}$. Consider the finitely many definable charts $\left\langle U_{i}, \phi_{i}\right\rangle$ 's for $G$ given by Pillay's theorem on definable groups in [20]. Then by construction, $U_{i}$ is a cell in $G \subseteq M^{k}$ of dimension $n$ or $U_{i}$ is a translate in $G$ of a cell in $G \subseteq M^{k}$ of dimension $n$. In the first case, $\phi_{i}$ is the restriction of a projection from $M^{k}$ onto some $n<k$ coordinates. In the second case $\phi_{i}$ is the composition of a translation in $G$ and the restriction of a projection as above. For the fact that the restriction of a projection as above is a definable homeomorphism see [2, Chapter $3,(2.7)]$.

Consider the open definable subsets $V_{i} \subseteq U_{i}(i=1, \ldots, l)$ given by Corollary 2.4 such that $\overline{V_{i}} \subseteq U_{i}$ and $\Omega=\bigcup_{i}\left(V_{i} \cap \Omega\right)$. It is enough to show that for every $U_{i}$ which is a cell in $G \subseteq M^{k}$, any definably compact definable subset $C$ of $G$ such that $C \subseteq U_{i} \subseteq M^{k}$ is bounded.

Fix $i$ such that $U_{i}$ is a cell in $G \subseteq M^{k}$ and suppose that $C$ is a definably compact definable subset of $G$ such that $C \subseteq U_{i}$ and $C$ is unbounded. Then there is a $j$ such that the projection of $C$ onto the $j$-coordinate is unbounded. Since $G$ has definable choice ([5, Theorem 7.2]) one of the following holds: (i) there is a definable map $\alpha:(e,+\infty) \subseteq M \longrightarrow U_{i} \subseteq G$ such that $\operatorname{im} \alpha \subseteq C$ and for each $t \in(e,+\infty)$, we have $\alpha_{j}(t)>t$ where $\alpha_{j}(t)$ is the $j$-coordinate of $\alpha(t)$; (ii) there is a definable map $\alpha:(-\infty, d) \subseteq M \longrightarrow U_{i} \subseteq G$ such that $\operatorname{im} \alpha \subseteq C$ and for each $t \in(-\infty, d)$, we have $\alpha_{j}(t)<t$ where $\alpha_{j}(t)$ is the $j$-coordinate of $\alpha(t)$. We assume (i) holds. For (ii) the proof is similar. By o-minimality we may assume that $\alpha$ is continuos with respect to the topology of $G$. Since $C$ is definably compact, the $\operatorname{limit}_{t \rightarrow+\infty} \alpha(t)$, with respect to the topology induced by $G$ on $C$, exists in $C$. Let $a$ be this limit.

By the observation in the first paragraph, the topology induced by $G$ on $U_{i}$ is the same as the topology induced by $M^{k}$ on $U_{i}$. Let $B$ be a bounded open box in $M^{k}$ containing $a$. Then $B \cap U_{i}$ is an open definable neighborhood of $a$ in $U_{i} \subseteq G$ in the topology of $G$. Thus there is a $t_{0} \in(e,+\infty)$ such that $\operatorname{im} \alpha_{\mid\left(t_{0},+\infty\right)} \subseteq B \cap U_{i} \subseteq B$. But this is absurd since $\operatorname{im} \alpha_{j \mid\left(t_{0},+\infty\right)}$ is unbounded.

For the rest of the section assume that $\mathcal{M}$ is a saturated o-minimal structure.

Let $G$ be a definable group defined over a small model $\mathcal{M}_{0}$ and let $Z$ be a definable subset of $G$, say defined over some parameter $a$ in $\mathcal{M}$. Thus, there is a uniformly $\mathcal{M}_{0}$-definable family $\{Z(t): t \in T\}$ of $\mathcal{M}_{0}$-definable subsets of $G$ such that $a \in T$ and $Z=Z(a)$. Let $\left\{Z^{\prime}(t): t \in T^{\prime}\right\}$ be another uniformly $\mathcal{M}_{0}$-definable family of $\mathcal{M}_{0}$-definable subsets of $G$ such that $a \in T^{\prime}$ and $Z=Z^{\prime}(a)$. Then the sets $\left\{t \in T: t=\operatorname{tp}\left(a / M_{0}\right)\right\}$ and $\left\{t \in T^{\prime}: t \models \operatorname{tp}\left(a / M_{0}\right)\right\}$ are equal (because $a \in T \cap T^{\prime}$ ) and the sub-families $\left\{Z(t): t \in T\right.$ and $\left.t=\operatorname{tp}\left(a / M_{0}\right)\right\}$ and $\left\{Z^{\prime}(t): t \in T^{\prime}\right.$ and $\left.t \mid=\operatorname{tp}\left(a / M_{0}\right)\right\}$ are equal (because $\left.Z(a)=Z=Z^{\prime}(a)\right)$. Recall that:

- the set of $\mathcal{M}_{0}$-conjugates of $Z$ is the same thing as the sub-family $\{Z(t)$ : $t \in T$ and $\left.t=\operatorname{tp}\left(a / M_{0}\right)\right\}$; 
- the set of $\mathcal{M}_{0}$-conjugates of $Z$ is finitely consistent if and only if the subfamily $\left\{Z(t): t \in T\right.$ and $\left.t \models \operatorname{tp}\left(a / M_{0}\right)\right\}$ has the finite intersection property (i.e., for any finite subset $S$ of $\left\{t \in T: t \models \operatorname{tp}\left(a / M_{0}\right)\right\}$ the intersection $\bigcap_{s \in S}\{Z(s): s \in S\}$ is non empty).

As explained in [17] and [14, Section 8] the crucial fact behind the theory of generic definable subsets of a definable group is the following result.

Theorem 3.2. Let $G$ be a definable group defined over a small model $\mathcal{M}_{0}$ and let $\Omega$ be a definably compact definable subset of $G$ defined over $\mathcal{M}_{0}$. If $X \subseteq G$ is a definable compact definable subset of $\Omega$, then the set of $\mathcal{M}_{0}$-conjugates of $X$ is finitely consistent if and only if $X$ has a point in $\mathcal{M}_{0}$.

Proof. Let $\left\langle U_{i}, \phi_{i}\right\rangle(i=1, \ldots, l)$ be the definable charts of $G$ given by Pillay's theorem on definable groups in [20]. Then the definable charts $\left\langle U_{i}, \phi_{i}\right\rangle(i=1, \ldots, l)$ are also defined over $\mathcal{M}_{0}$. By Lemma 3.1, there are open definable subsets $V_{i} \subseteq U_{i}$ of $G$, with $i=1, \ldots, l$, such that $\overline{V_{i}} \subseteq U_{i}, \Omega=\bigcup_{i}\left(V_{i} \cap \Omega\right)=\bigcup_{i}\left(\overline{V_{i}} \cap \Omega\right)$ and, for each $i, \phi_{i}\left(\overline{V_{i}} \cap \Omega\right)$ is a closed and bounded definable subset of $M^{n}$ where $n=\operatorname{dim} G$. Moreover, if for each $i$ we set $X_{i}=\overline{V_{i}} \cap \Omega \cap X \subseteq U_{i}$, then $X=\bigcup_{i} X_{i} \subseteq \Omega, \phi_{i}\left(X_{i}\right)$ is a closed and bounded definable subset of $\phi_{i}\left(U_{i}\right)$ and $\phi_{i}\left(U_{i}\right)$ has definable choice since the same is true for $U_{i} \subseteq G$.

Let $a$ be a parameter in $\mathcal{M}$ over which $X$ is defined. Consider a uniformly $\mathcal{M}_{0}$-definable family $\{X(t): t \in T\}$ of $\mathcal{M}_{0}$-definable subsets of $G$ such that $a \in T$ and $X=X(a)$. Since $X \subseteq \Omega$ and $\Omega$ is defined over $\mathcal{M}_{0}$, after replacing $T$ by an $\mathcal{M}_{0}$-definable subset, we may assume that for all $t \in T$ we have $X(t) \subseteq \Omega$. Since "closed" is a first-order property, we may also assume that $X(t)$ is a closed (hence definably compact) definable subset of $\Omega$ for all $t \in T$. If for each $i$ and $t \in T$ we set $X_{i}(t)=\bar{V}_{i} \cap \Omega \cap X(t) \subseteq U_{i}$, then we get uniformly $\mathcal{M}_{0}$-definable families $\left\{X_{i}(t): t \in T\right\}$ of $\mathcal{M}_{0}$-definable subsets of $G$ such that $X(t)=\bigcup_{i} X_{i}(t) \subseteq \Omega$ and $\phi_{i}\left(X_{i}(t)\right)$ is a closed and bounded definable subset of $\phi_{i}\left(U_{i}\right)$.

Recall that the set of $\mathcal{M}_{0}$-conjugates of $X$ is the sub-family $\{X(t): t \in T$ and $\left.t \models \operatorname{tp}\left(a / M_{0}\right)\right\}$, and for each $i$, the set of $\mathcal{M}_{0}$-conjugates of $X_{i}$ (resp. $\left.\phi_{i}\left(X_{i}\right)\right)$ is the sub-family $\left\{X_{i}(t): t \in T\right.$ and $\left.t \models \operatorname{tp}\left(a / M_{0}\right)\right\}$ (resp. $\left\{\phi_{i}\left(X_{i}(t)\right): t \in T\right.$ and $\left.\left.t=\operatorname{tp}\left(a / M_{0}\right)\right\}\right)$.

Note also that by the remarks before the proof of [17, Theorem 2.1] (in the Appendix of that paper) together with its proof we can use this theorem to conclude that: the set of $\mathcal{M}_{0}$-conjugates of $\phi_{i}\left(X_{i}\right)$ is finitely consistent if and only if $\phi_{i}\left(X_{i}\right)$ has a point in $\mathcal{M}_{0}$. Since each $\phi_{i \mid X_{i}}: X_{i} \longrightarrow \phi_{i}\left(X_{i}\right)$ is the restriction of a definable bijection $\phi_{i}$ defined over $\mathcal{M}_{0}$, we have that $\mathcal{M}_{0}$-conjugation commutes with $\phi_{i}$ and with $\phi_{i}^{-1}$ and the image by $\phi_{i}$ and by $\phi_{i}^{-1}$ of a point in $\mathcal{M}_{0}$ is a point in $\mathcal{M}_{0}$. Hence, the set of $\mathcal{M}_{0}$-conjugates of $X_{i}$ is finitely consistent if and only if $X_{i}$ has a point in $\mathcal{M}_{0}$.

It is obvious that if $X$ has a point in $\mathcal{M}_{0}$, then the set of $\mathcal{M}_{0}$-conjugates of $X$ is finitely consistent. Conversely suppose that the set of $\mathcal{M}_{0}$-conjugates of $X$ is finitely consistent. By the observation in the previous paragraph it is enough to show that for some $i$, the set of $\mathcal{M}_{0}$-conjugates of $X_{i}$ is finitely consistent. By our assumption, the collection $\left\{X(t): t \in T\right.$ and $\left.t=\operatorname{tp}\left(a / M_{0}\right)\right\}$ of definable subsets of $G$ is a partial type which extends to a complete type $p$ over $M$. Let $b=p$ in some $|M|^{+}$-saturated elementary extension $\mathcal{M}^{\prime}$ of $\mathcal{M}$. Then $b \in \bigcap\left\{X(t)\left(M^{\prime}\right): t \in T\right.$ and 
$\left.t \models \operatorname{tp}\left(a / M_{0}\right)\right\} \subseteq \Omega\left(M^{\prime}\right)$. So there is some $i$ such that $b \in \overline{V_{i}}\left(M^{\prime}\right) \cap \Omega\left(M^{\prime}\right)$ (since $\left.\Omega=\bigcup_{i}\left(\overline{V_{i}} \cap \Omega\right)\right)$ and consequently, $b \in \bigcap\left\{X_{i}(t)\left(M^{\prime}\right): t \in T\right.$ and $\left.t \models \operatorname{tp}\left(a / M_{0}\right)\right\}$ (since $X_{i}(t)=\overline{V_{i}} \cap \Omega \cap X(t)$ by definition). Thus, as required, we found an $i$ such that the set of $\mathcal{M}_{0}$-conjugates of $X_{i}$ is finitely consistent.

We can now state the main result on generic definable subsets of a definably compact definable group $G$ which can be proved from Theorem 3.2 just like [17, Theorem 3.6]. Recall that a definable subset $X$ of $G$ is left (resp. right) generic if finitely many left (resp. right) translates of $X$ by elements of $G$ cover $G$.

Theorem 3.3. Assume that $G$ is a definably compact definable group and $X \subseteq G$ is a definable subset. If $X$ is not left generic, then $G \backslash X$ is right generic.

Proof. We may assume that both $G$ and $X$ are defined over a small model $\mathcal{M}_{0}$. By [17, Lemma 3.4 (ii)], we may assume that $X$ is a closed definable subset of $G$. Since $X$ is not left generic, for every $h_{1}, \ldots, h_{k} \in G$ there is $g \in G$ such that $h_{i} \notin X g$, for $i=1, \ldots, k$. By first-order logic compactness, there is $g \in G$ such that $X g$ has no point in $\mathcal{M}_{0}$. So by Theorem 3.2, the set of $\mathcal{M}_{0}$-conjugates of the definably compact definable subset $X g$ of $G$ (which is definably compact and defined over the small model $\mathcal{M}_{0}$ ) is not finitely consistent. This means that the family $\left\{X g^{\prime}: g^{\prime} \models \operatorname{tp}\left(g / M_{0}\right)\right\}$ does not have the finite intersection property. Therefore, there are $g_{1}, \ldots, g_{l} \in G$ all realizing $\operatorname{tp}\left(g / M_{0}\right)$ such that $X g_{1} \cap \cdots \cap X g_{l}=\emptyset$. In particular, $G \backslash X$ is right generic as required.

From Theorem 3.3 we obtain just like in [17, Section 3]:

Theorem 3.4. If $G$ is a definably compact, abelian, definable group, then:

- the union of two non generic definable subsets of $G$ is also non generic;

- there is a complete generic type of $G$ (whose formulas define generic definable subsets),

- for every definable subset $X$ of $G$ its stabilizer $\operatorname{Stab}(X)=\{g \in G$ : $X \Delta(g X)$ is non generic $\}$ is a type definable subgroup of $G$.

Also from Theorem 3.3 we obtain just like in [17, Section 4]:

Theorem 3.5. Let $G$ be a definably compact definable group and $S \subseteq G$ a definable semi-group in $G$ (i.e., a definable set closed under the group operation of $G$ ). If the closure of $S$ in $G$ is definably compact, then $S$ is a subgroup of $G$. In particular, if $G$ is definably compact, then every definable semi-group in $G$ is a subgroup of $G$.

\section{UNIFORM DEFINABILITY OF DEFINABLE COMPACTNESS}

Here we show that the notion of "definably compact group" is a first-order notion in arbitrary o-minimal structures, i.e., if $\left\{G_{s}: s \in S\right\}$ is a uniformly definable family of definable groups, then the set of $s$ for which $G_{s}$ is definably compact is definable. In the special case of o-minimal expansions of ordered groups this is [14, Lemma 7.4].

Lemma 4.1. Let $\left\{G_{s}: s \in S\right\}$ be a uniformly definable family of definable groups in $M^{k}$. If $\left\{C_{s}: s \in S\right\}$ is uniformly definable sub-family of definable subsets, then there exists a definable map $f: S \longrightarrow \bigcup_{s \in S} G_{s}$ such that $f(s) \in C_{s}$ for each $s \in S$. 
Proof. Suppose that the families $\left\{G_{s}: s \in S\right\}$ and $\left\{C_{s}: s \in s\right\}$ are defined over $A$. Since each $G_{s}$ has definable choice ([5, Theorem 7.2]), for each $s \in S$, there exists a definable element $c_{s} \in C_{s}$ defined over $\{s\} \cup A$. Let $\psi_{s}\left(x, y, a_{s}\right)$ be a first-order formula over $A$ such that $\psi_{s}\left(x, s, a_{s}\right)$ defines $c_{s}$. For each $s \in S$, let $B_{s}$ be the subset of $S$ of all $t \in S$ such that $\psi_{s}\left(x, t, a_{s}\right)$ defines an element of $C_{t}$. Then $S=\bigcup_{s \in S} B_{s}$ and each $B_{s}$ is a definable set defined over $A$. Since $S$ is also defined over $A$, by first-order logic compactness, there are $s_{0}, \ldots, s_{l} \in S$ such that $S=B_{s_{0}} \cup \cdots \cup B_{s_{l}}$. Take a cell decomposition $\mathcal{C}$ of $S$ compatible with the definable subsets $B_{s_{0}}, \ldots, B_{s_{l}}$ and for each $C \in \mathcal{C}$ choose $s_{C} \in\left\{s_{i}: C \subseteq B_{s_{i}}\right.$ and $\left.i=0, \ldots, l\right\}$. Consider the definable map $f: S \longrightarrow \bigcup_{s \in S} G_{s}$ defined over $A$ such that for $C \in \mathcal{C}, f_{\mid C}$ is defined by the formula $\psi_{s_{C}}\left(x, y, a_{s_{C}}\right)$. Then $f(s) \in C_{s}$ for each $s \in S$ as required.

Notation: Let $\left\{G_{s}: s \in S\right\}$ be a uniformly definable family of definable groups in $M^{k}$ with $\operatorname{dim} G_{s}=n$ for all $s \in S$. By Pillay's theorem on definable groups in [20] there exists, uniformly in $s$, a definable family of finitely many definable charts $\left\{\left\langle U_{i, s}, \phi_{i, s}\right\rangle: s \in S, i=1, \ldots, l\right\}$ for the $G_{s}$ 's. For each $s \in S$, let $e_{G_{s}}$ be the identity element of $G_{s}$. Since, for each $s \in S$ we can definably choose $i_{s} \in\{1, \ldots, l\}$ such that $e_{G_{s}} \in U_{i_{s}, s}$, we may assume that for all $s \in S$, we have $e_{G_{s}} \in U_{s}=U_{i, s}$ for some fixed $i$. Set $\phi_{s}=\phi_{i, s}$. Let $\phi_{s}\left(e_{G_{s}}\right)=\left\langle e_{1, s}, \ldots, e_{n, s}\right\rangle$. Since for each $j=1, \ldots, n$, there exists $J_{j, s}=\left(d_{j, s}^{-}, d_{j, s}^{+}\right) \subseteq M$ an open (definable) interval such that $e_{j, s} \in J_{j, s}$ and $\Pi_{j=1}^{n} J_{j, s} \subseteq \phi_{s}\left(U_{s}\right)$, by Lemma 4.1, we can assume that $\left\{J_{j, s}: s \in S\right\}$ is uniformly definable. Let $O_{s}=\phi_{s}^{-1}\left(\Pi_{j=1}^{n} J_{j, s}\right)$ which is an open definable neighborhood of $e_{G_{s}}$ in $G_{s}$. Then $\left\{O_{s}: s \in S\right\}$ is a uniformly definable sub-family of $\left\{G_{s}: s \in S\right\}$.

For each $s \in S$ and $j=1, \ldots, n$, let $J_{j, s}^{-}=\left\{x \in J_{j, s}: x<e_{j, s}\right\}$ and $J_{j, s}^{+}=\{x \in$ $\left.J_{j, s}: e_{j, s}<x\right\}$. For $\delta_{s}=\left\langle\delta_{1, s}^{-}, \delta_{1, s}^{+}, \ldots, \delta_{n, s}^{-}, \delta_{n, s}^{+}\right\rangle \in \Pi_{j=1}^{n}\left(J_{j, s}^{-} \times J_{j, s}^{+}\right)$, let

$$
O_{s}^{\delta_{s}}=\left\{x \in O_{s}: \phi_{s}(x)=\left\langle z_{1}, \ldots, z_{n}\right\rangle \text { and } \delta_{j, s}^{-}<z_{j}<\delta_{j, s}^{+} \text {for all } j\right\}
$$

and

$$
{\overline{O_{s}}}^{\delta_{s}}=\left\{x \in O_{s}: \phi_{s}(x)=\left\langle z_{1}, \ldots, z_{n}\right\rangle \text { and } \delta_{j, s}^{-} \leq z_{j} \leq \delta_{j, s}^{+} \text {for all } j\right\} .
$$

If $\left\{D_{s}: s \in S\right\}$ is a uniformly definable sub-family of $\left\{G_{s}: s \in S\right\}$, we consider

$$
O_{s}^{\delta_{s}}\left(D_{s}\right)=\cup\left\{d O_{s}^{\delta_{s}}: d \in D_{s}\right\}
$$

and

$$
{\overline{O_{s}}}^{\delta_{s}}\left(D_{s}\right)=\cup\left\{d{\overline{O_{s}}}^{\delta_{s}}: d \in D_{s}\right\} \text {. }
$$

We have that, for each $s \in S, O_{s}^{\delta}\left(D_{s}\right)$ is an open definable neighborhood of $D_{s}$ in $G_{s}$ and $O_{s}^{\delta}\left(D_{s}\right) \subseteq{\overline{O_{s}}}^{\delta}\left(D_{s}\right)$.

Proposition 4.2. Let $\left\{G_{s}: s \in S\right\}$ be a uniformly definable family of definable groups in $M^{k}$ and $\left\{K_{s}: s \in S\right\}$ a uniformly definable sub-family of definable subsets. Then the set of $s$ for which $K_{s}$ is a definably compact definable subset of $G_{s}$ is definable.

Proof. By definability of dimension ([20]) we may assume without loss of generality that each $G_{s}$ has dimension $n$. Let $\left\{\left\langle U_{i, s}, \phi_{i, s}\right\rangle: s \in S, i=1, \ldots, l\right\}$ be a uniformly definable family of finitely many definable charts for the $G_{s}$ 's. 
Consider the subset $S^{\prime}$ of $S$ of all $s \in S$ such that the following condition holds: $\forall \delta_{1, s} \ldots \forall \delta_{l, s}\left[\left(\bigwedge_{i=1}^{l} \delta_{i, s} \in \Pi_{j=1}^{n}\left(J_{j, s}^{-} \times J_{j, s}^{+}\right)\right) \Rightarrow\left[\bigvee_{i=1}^{l}\left(\phi_{i, s}\left(\overline{O_{s}^{\delta_{i, s}}\left(A_{i, s}\right)} \cap K_{s}\right)\right.\right.\right.$ is unbounded or $K_{s} \nsubseteq \bigcup_{i=1}^{l} O_{s}^{\delta_{i, s}}\left(A_{i, s}\right)$ or $\left.\bigvee_{i=1}^{l}\left(O_{s}^{\delta_{i, s}}\left(A_{i, s}\right) \nsubseteq U_{i, s}\right)\right]$, where the $A_{i, s}$ 's are given inductively by

$$
A_{i, s}=K_{s} \backslash\left(\bigcup_{m<i} O_{s}^{\delta_{m, s}}\left(A_{m, s}\right) \cup \bigcup_{j=i+1}^{l} U_{j, s}\right) .
$$

By definability of "bounded" in $M^{k}, S^{\prime}$ is a definable subset of $S$. So by Lemma 3.1, after replacing $S$ by $S \backslash S^{\prime}$, we may assume that for all $s \in S$ the following first-order condition holds:

$\exists \delta_{1, s} \ldots \exists \delta_{l, s}\left[\left(\bigwedge_{i=1}^{l} \delta_{i, s} \in \Pi_{j=1}^{n}\left(J_{j, s}^{-} \times J_{j, s}^{+}\right)\right) \wedge\left[\bigwedge_{i=1}^{l}\left(\phi_{i, s} \overline{\left(O_{s}^{\delta_{i, s}}\left(A_{i, s}\right)\right.} \cap K_{s}\right)\right.\right.$ is bounded and $K_{s} \subseteq \bigcup_{i=1}^{l} O_{s}^{\delta_{i, s}}\left(A_{i, s}\right)$ and $\left.\bigwedge_{i=1}^{l}\left(O_{s}^{\delta_{i, s}}\left(A_{i, s}\right) \subseteq U_{i, s}\right)\right]$.

By Lemma 4.1, there exist definable maps $\delta_{1}, \ldots, \delta_{l}: S \longrightarrow \Pi_{j=1}^{n}\left(J_{j, s}^{-} \times J_{j, s}^{+}\right)$such that for all $s \in S, \bigwedge_{i=1}^{l}\left(\phi_{i, s}\left(\overline{O_{s}^{\delta_{i}(s)}\left(A_{i, s}\right)} \cap K_{s}\right)\right.$ is bounded and $K_{s} \subseteq \bigcup_{i=1}^{l} O_{s}^{\delta_{i}(s)}\left(A_{i, s}\right)$ and $\bigwedge_{i=1}^{l}\left(O_{s}^{\delta_{i}(s)}\left(A_{i, s}\right) \subseteq U_{i, s}\right)$. For each $i=1, \ldots, l$ and $s \in S$, set $W_{i, s}=O^{\delta_{i}(s)}\left(A_{i, s}\right) \cap K_{s}$ and if $\epsilon_{s} \in \Pi_{j=1}^{n}\left(J_{j, s}^{-} \times J_{j, s}^{+}\right)$, let

$$
W_{i, s}^{\epsilon_{s}}=W_{i, s} \backslash \bar{O}_{s}^{\epsilon_{s}}\left(\overline{W_{i, s}} \backslash W_{i, s}\right) .
$$

Claim 4.3. For each $s \in S$, the definable set $K_{s}$ is definably compact in $G_{s}$ if and only if there exists $\epsilon_{s} \in \Pi_{j=1}^{n}\left(J_{j, s}^{-} \times J_{j, s}^{+}\right)$such that

$$
K_{s}=\bigcup_{i=1}^{l} W_{i, s}^{\epsilon_{s}} .
$$

Proof. Fix $s$, and assume that $K_{s}$ is definably compact in $G_{s}$. For each $i=1, \ldots, l$, let $V_{i, s}$ be open definable subsets of $G_{s}$ such that $\overline{V_{i, s}} \subseteq W_{i, s}$ and $K_{s}=\bigcup_{i=1}^{l}\left(V_{i, s} \cap K_{s}\right)$ (Corollary 2.4). Then $\overline{V_{i, s}} \cap\left(\overline{W_{i, s}} \backslash W_{i, s}\right)=\emptyset$ and so $K_{s} \backslash \overline{V_{i, s}}$ is an open definable neighborhood of $\overline{W_{i, s}} \backslash W_{i, s}$ in $K_{s}$. Since $\overline{W_{i, s}} \backslash W_{i, s}$ is definably compact, it follows from Proposition 2.2, that there exists $\epsilon_{s} \in \Pi_{j=1}^{n}\left(J_{j, s}^{-} \times J_{j, s}^{+}\right)$ such that $K_{s}=\bigcup_{i=1}^{l} W_{i, s}^{\epsilon_{s}}$. For the converse, if there is an $\epsilon_{s}$ as above, then any (continuous) definable curve $\alpha$ in $K_{s}$ will be eventually contained in one of the $W_{i, s}^{\epsilon_{s}}$. Since $\phi_{i, s}\left(W_{i, s}^{\epsilon_{s}}\right)$ is bounded the (continuous) definable curve $\phi_{i, s} \circ \alpha$ has a limit $a \in M^{n}$, which must be in $\phi_{i, s}\left(U_{i, s}\right)$. Thus $\phi_{i, s}^{-1}(a) \in K_{s}$ is the limit of $\alpha$.

We end the section with an observation about another uniform definability result from [14]. In [14, Lemma 7.4 (ii)] it is proved that in an o-minimal expansion of an ordered group, if $\left\{G_{s}: s \in S\right\}$ is a uniformly definable family of abelian definable groups, then the set of $s$ for which $G_{s}$ is definably connected is definable. We point out that the proof there is very general and the above assumptions are not necessary. Hence:

Remark 4.4. If $\left\{G_{s}: s \in S\right\}$ is a uniformly definable family of definable groups, then the set of $s$ for which $G_{s}$ is definably connected is definable.

\section{REFERENCES}

[1] A. Berarducci, M. Otero, Y. Peterzil and A. Pillay A descending chain condition for groups definable in o-minimal structures Ann. Pure Appl. Logic 134 (2005) 303 -313 . 
[2] L. van den Dries Tame topology and o-minimal structures Cambridge University Press 1998.

[3] A. Dolich Forking and independence in o-minimal theories J. Symb. Logic 69 (2004) $215-240$.

[4] M. Edmundo Structure theorems for o-minimal expansions of groups Ann. Pure Appl. Logic 102 (1-2) (2000) 159 - 181.

[5] M. Edmundo Solvable groups definable in o-minimal structures J. Pure Appl. Algebra 185 (1-3) (2003) 103 - 145.

[6] M. Edmundo, G. Jones and N. Peatfield Sheaf cohomology in o-minimal structures J. Math. Logic 6 (2) (2006) 163 - 179.

[7] M. Edmundo and M. Otero Definably compact abelian groups J. Math. Logic 4 (2) (2004) $163-180$

[8] M. Edmundo and N. Peatfield O-minimal Čech cohomology Quart. J. Math. 59 (2) (2008) $213-220$

[9] M. Edmundo and L. Prelli Poincaré - Verdier duality in o-minimal structures Ann. Inst. Fourier Grenoble 60 (4) (2010) 1259 - 1288.

[10] M. Edmundo and A. Woerheide Comparation theorems for o-minimal singular (co)homology Trans. Amer. Math. Soc. 360 (9) (2008) 4889 - 4912.

[11] P. Eleftheriou A semi-linear group which is not affine Ann. Pure Appl. Logic 156 (2008) $287-289$

[12] P. Eleftheriou and S. Starchenko Groups definable in ordered vector spaces over ordered division rings J. Symb. Logic 72 (2007) 1108 - 1140.

[13] E. Hrushovski, Y. Peterzil and A. Pillay Groups, measures and the NIP J. Amer. Math. Soc. 21 (2) (2008) 563 - 596.

[14] Y. Peterzil Returning to semi-bounded sets J. Symb. Logic 74 (2) (2009) $597-617$.

[15] Y. Peterzil and C. Steinhorn Definable compacteness and definable subgroups of o-minimal groups J. London Math. Soc. 59 (2) (1999) 769 - 786.

[16] Y. Peterzil and S. Starchenko Definable homomorphisms of abelian groups definable in o-minimal structures Ann. Pure Appl. Logic 101 (1) (1999) 1 - 27.

[17] Y. Peterzil and A. Pillay Generic sets in definably compact groups Fund. Math. 193 (2) (2007) 153-170.

[18] Y. Peterzil, A. Pillay and S. Starchenko Definably simple groups in o-minimal structures Trans. Amer. Math. Soc. 352 (10) (2000) 4397 - 4419.

[19] Y. Peterzil, A. Pillay and S. Starchenko Linear groups definable in o-minimal structures J. Algebra 247 (2002) $1-23$.

[20] A. Pillay On groups and fields definable in o-minimal structures J. Pure Appl. Algebra 53 (1988) 239 - 255.

[21] A. Pillay Type-definability, compact Lie groups and o-minimality J. Math. Logic 4 (2) (2004) $147-162$.

[22] A. Woerheide O-minimal homology PhD. Thesis (1996), University of Illinois at Urbana-Champaign.

Universidade Aberta and CMaf Universidade de Lisboa, Av. Prof. Gama Pinto 2, 1649-003 Lisboa, Portugal

E-mail address: edmundo@cii.fc.ul.pt

Department of Mathematics, Seconda Università di Napoli, Via Vivaldi 43, 81100 CASerta, Italy

E-mail address: giuseppina.terzo@unina2.it 